\title{
HOOPS AND THEIR IMPLICATIONAL REDUCTS
}

\section{(ABSTRACT)}

\author{
W. J. BLOK \\ Department of Mathematics, Statistics and Computer Science \\ University of Illinois at Chicago, Box 4348, Chicago, Illinois 60680, U.S.A. \\ E-mail:U47565@UICVM.BITNET,U47565@UICVM.UIC.EDU
}

\section{M. A. FERREIRIM}

Departamento de Matemática, Faculdade de Ciências

Universidade de Lisboa, Lisbon, Portugal

E-mail: MIMAFER@PTEARN.BITNET

1. Preliminaries. A structure $\mathbf{A}=\langle A, \cdot, 1, \leq\rangle$ is a partially ordered monoid if $\langle A, \cdot, 1\rangle$ is a monoid, $\leq$ is a partial order on $A$, and for all $x, y, z \in A$, if $x \leq y$, then $x \cdot z \leq y \cdot z$ and $z \cdot x \leq z \cdot y$. A is integral if $x \leq 1$, for all $x \in A$. Finally, $\mathbf{A}$ is residuated if for all $x, y \in A$ the set $\{z: z \cdot x \leq y\}$ contains a largest element, called the residual of $x$ relative to $y$, and denoted by $x \rightarrow y$. A partially ordered commutative, residuated and integral monoid $\langle A, \cdot, 1, \leq\rangle$ can be treated as an algebra $\langle A, \cdot, \rightarrow, 1\rangle$, since the partial order $\leq$ can be recovered via $x \leq y$ iff $x \rightarrow y=1$. We will refer to the algebras thus obtained by the acronym pocrim. The class $\mathbb{M}$ of all pocrims is a quasivariety definable by:

(M1) $\quad x \cdot 1 \approx x$,

(M2) $\quad x \cdot y \approx y \cdot x$,

(M3) $x \rightarrow 1 \approx 1$,

(M4) $1 \rightarrow x \approx x$,

(M5) $\quad(x \rightarrow y) \rightarrow((z \rightarrow x) \rightarrow(z \rightarrow y)) \approx 1$,

(M6) $\quad x \rightarrow(y \rightarrow z) \approx(x \cdot y) \rightarrow z$,

(M7) $\quad x \rightarrow y \approx 1 \& y \rightarrow x \approx 1 \Rightarrow x \approx y$.

1991 Mathematics Subject Classification: 06F05, 06F20, 03G20, 06F35. 
Higgs [11] shows that $\mathbb{M}$ is not a variety.

The class $\mathbb{B} \mathbb{C K}$ of BCK-algebras consists of all algebras $\mathbf{A}=\langle A, \rightarrow, 1\rangle$ satisfying (M3), (M4), (M5) and (M7) together with

(M8) $\quad x \rightarrow x \approx 1$,

(M9) $\quad x \rightarrow(y \rightarrow z) \approx y \rightarrow(x \rightarrow z)$.

Since (M8) and (M9) are satisfied in $\mathbb{M}$ the class of $\{\rightarrow, 1\}$-subreducts of algebras in $\mathbb{M}$ consists of BCK-algebras; in symbols, $\mathbf{S}\left(\mathbb{M}^{\{\rightarrow, 1\}}\right) \subseteq \mathbb{B} \mathbb{C}$. The reverse inclusion also holds; this was shown independently by Pałasiński [15], Ono and Komori [14], and Fleischer [9]. Wroński [18] and Higgs [11] show that $\mathbb{B C K}$ is not a variety.

A partially ordered monoid $\mathbf{A}=\langle A, \cdot, 1, \leq\rangle$ is naturally ordered if for all $x, y$ $\in A$,

$$
x \leq y \text { if and only if there is a } z \in A \text { such that } x=z \cdot y \text {. }
$$

A hoop is a pocrim that is naturally ordered; we will denote the class of hoops by $\mathbb{H O}$. Bosbach [4] shows this class is a variety: an algebra $\langle A, \cdot, \rightarrow, 1\rangle$ is a hoop if and only if $\langle A, \cdot, 1\rangle$ is a commutative monoid that satisfies the following identities:

(M8) $\quad x \rightarrow x \approx 1$,

(M6) $\quad x \rightarrow(y \rightarrow z) \approx(x \cdot y) \rightarrow z$,

$\left(\mathrm{H}^{\prime}\right) \quad(x \rightarrow y) \cdot x \approx(y \rightarrow x) \cdot y$.

The variety of hoops was further studied by Büchi and Owens [5] in the midseventies, but their work was not completed and never published. Their unpublished manuscript is rich in ideas, and a number of these played an essential role in the discovery of our results.

The variety of hoops includes two classes of algebras that are closely related to familiar algebras of logic: the varieties of Brouwerian semilattices and the variety of Wajsberg hoops. Brouwerian semilattices are the $\{\wedge, \rightarrow, 1\}$-subreducts of Heyting algebras, which are the algebraic models of intuitionistic propositional logic. Wajsberg hoops are the $\{\cdot, \rightarrow, 1\}$-subreducts of Wajsberg algebras, which are the algebraic models of Łukasiewicz's many-valued logic. The class of hoops is, in a sense we will make precise (see Theorem 3.3 and the discussion following it), a natural common generalization of the varieties of Brouwerian semilattices and Wajsberg hoops. Or, phrased in terms of logics, the class of hoops is the algebraic semantics of an algebraizable deductive system (in the sense of [2]) that embodies the $\{\cdot, \rightarrow, 1\}$-fragments of intuitionistic propositional logic as well as Łukasiewicz's many-valued logic, and might be viewed as an intuitionistic version of Łukasiewicz's logic.

The main aim of the paper is to show that the variety of hoops is generated, as a quasivariety, by its finite members (Corollary 3.6.) The proof rests on an analysis of the structure of the subdirectly irreducible hoops, which allows us to reduce the problem to the question whether the variety of Wajsberg hoops is 
generated, as a quasivariety, by its finite members. In Section 2 we establish that this is indeed the case (Theorem 2.5).

Since $\mathbb{H} \mathbb{O} \subseteq \mathbb{M}$, the class $\mathbf{S}(\mathbb{H} \mathbb{O}\{\rightarrow, 1\})$ of $\{\rightarrow, 1\}$-subreducts of hoops is a quasivariety of BCK-algebras satisfying the following implicational consequence of the hoop axiom $\left(\mathrm{H}^{\prime}\right)$ :

$$
(x \rightarrow y) \rightarrow(x \rightarrow z) \approx(y \rightarrow x) \rightarrow(y \rightarrow z) .
$$

In Section 4 we confirm a conjecture by Wroński ([17]) which says that this identity, together with the BCK-axioms, actually defines $\mathbf{S}\left(\mathbb{H} \mathbb{O}^{\{\rightarrow, 1\}}\right)$. The model theory of this class can be developed along the same lines as that of the class of hoops, and yields that it is a variety as well; this answers a problem proposed in $[17]$.

For the basic properties of hoops we refer to [3]. In particular, every hoop is a meet-semilattice, with the meet operation $\wedge$ definable by $x \wedge y:=(x \rightarrow y) \cdot x$. If $\mathbf{A}$ is a hoop, then $F \subseteq A$ is a filter of $\mathbf{A}$ if $1 \in F, F$ is closed under $\cdot$, and $F$ is upward closed. The map $\Theta \mapsto 1 / \Theta$ establishes an isomorphism between the lattice of congruences of $\mathbf{A}$ and its lattice of filters; the inverse of this map is

$$
F \mapsto\{(x, y):(x \rightarrow y) \cdot(y \rightarrow x) \in F\} .
$$

If $F$ is the filter associated with the congruence $\Theta$, we often write $\mathbf{A} / F$ for $\mathbf{A} / \Theta$. Observe that a hoop is subdirectly irreducible if and only if it possesses a filter $U \neq\{1\}$ that is contained in every non-trivial filter of the hoop. The variety $\mathbb{H} \mathbb{O}$ is congruence distributive and congruence permutable.

2. Wajsberg hoops. Wajsberg algebras are the algebraic models of Łukasiewicz's $\aleph_{0}$-valued logic. In [3] it is shown that the variety of Wajsberg algebras is term equivalent to the variety of algebras $\langle A, \cdot, \rightarrow, 0,1\rangle$ satisfying:

(W1) $\langle A, \cdot, \rightarrow, 1\rangle$ is a hoop,

(W2) $\quad 0 \rightarrow x \approx 1$,

(T) $\quad(x \rightarrow y) \rightarrow y \approx(y \rightarrow x) \rightarrow x$.

These algebras are actually (bounded) lattices, with join operation

$$
x \vee y:=(x \rightarrow y) \rightarrow y .
$$

The identity $(\mathrm{T})$ thus expresses the fact $\vee$ is a commutative operation, and is therefore traditionally referred to as the commutative law. A Wajsberg hoop is a hoop that satisfies the commutative law $(\mathrm{T})$. We denote the class of Wajsberg hoops by $\mathbb{W} \mathbb{H} \mathbb{O}$. Note that the bounded Wajsberg hoops are exactly the $\{\cdot, \rightarrow, 1\}$ reducts of Wajsberg algebras.

If $\mathbf{A}$ is a Wajsberg hoop, and $a \in A$, let $A_{a}=\{x \in A: x \geq a\}$. Since $x \rightarrow y \geq y, A_{a}$ is closed under $\rightarrow$. Define for $x, y \in A_{a}$,

$$
x \cdot{ }_{a} y=(x \cdot y) \vee a,
$$


and set $\mathbf{A}_{a}=\left\langle A_{a},{ }^{\cdot}, \rightarrow, 1\right\rangle$. Then $\mathbf{A}_{a}$ is a bounded Wajsberg hoop, and hence a reduct of a Wajsberg algebra. We have

Lemma 2.1. Let $\mathbf{A}$ be a Wajsberg hoop. Then $\mathbf{A} \in \mathbf{I S P}_{U}\left(\left\{\mathbf{A}_{a}: a \in A\right\}\right)$.

We may conclude:

Proposition 2.2. The variety $\mathbb{W H} \mathbb{O}$ of Wajsberg hoops coincides with the class of $\{\cdot, \rightarrow, 1\}$-subreducts of Wajsberg algebras.

There is a close connection between Abelian lattice-ordered groups (Abelian $l$-groups for short) and Wajsberg hoops. If $\mathbf{G}=\langle G,+,-, 0, \vee, \wedge\rangle$ is an Abelian $l$-group, the positive cone $P(\mathbf{G})$ of $\mathbf{G}$ is the set $\{x \in G: x \vee 0=x\}$. Let $\mathbf{P}(\mathbf{G})$ be the algebra $\langle P(\mathbf{G}), \cdot, \rightarrow, 1\rangle$, with $x \cdot{ }^{\mathbf{P}(\mathbf{G})} y=x+{ }^{\mathbf{G}} y, x \rightarrow^{\mathbf{P}(\mathbf{G})} y=\left(y-{ }^{\mathbf{G}} x\right) \vee^{\mathbf{G}} 0$

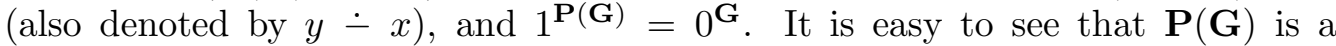
hoop; note that the partial order of $\mathbf{P}(\mathbf{G})$ is the converse of the partial order inherited from $\mathbf{G}$. In addition, $\mathbf{P}(\mathbf{G})$ satisfies the commutative law $(\mathrm{T})$, and also the cancellative law:

$$
y \rightarrow(y \cdot x) \approx x .
$$

Observe that any hoop satisfying $(\mathrm{C})$ is cancellative as a monoid: if $y \cdot x=y \cdot z$, then $x=z$. We can now appeal to a classical result (see for example $[1, \mathrm{p} .321]$ ) to see that if $\mathbf{A}$ is a hoop satisfying $(\mathrm{C})$, then there is an Abelian $l$-group $\mathbf{G}$ such that $\mathbf{A} \cong \mathbf{P}(\mathbf{G})$. In fact, we have

Theorem 2.3. The functor $\mathbf{G} \mapsto \mathbf{P}(\mathbf{G}),\left.f \mapsto f\right|_{P(\mathbf{G})}$ establishes an equivalence between the category of Abelian l-groups and l-homomorphisms on the one hand and the category of cancellative hoops and homomorphisms on the other.

Let $\mathbf{Z}$ denote the usual $l$-group of integers $\langle Z,+,-, 0, \vee, \wedge\rangle$, and let $\mathbf{C}_{\infty}$ denote the algebra $\left\langle\left\{1=a^{0}, a, a^{2}, \ldots\right\}, \cdot, \rightarrow, 1\right\rangle$, with $a^{n} \cdot a^{m}=a^{n+m}$, and $a^{n} \rightarrow a^{m}=$ $a^{m \dot{-} n}$. Note that $\mathbf{C}_{\infty}$ is isomorphic to $\mathbf{P}(\mathbf{Z})$.

Corollary 2.4. The variety of cancellative hoops equals $\mathbf{I S P P} \mathbf{P}_{U}\left(\mathbf{C}_{\infty}\right)$.

P r o of. It is well known (see for example [12]) that the variety of Abelian $l$ groups equals $\mathbf{I S P P}_{U}(\mathbf{Z})$. The result follows from the fact the equivalence functor of the previous theorem commutes with the operators $\mathbf{I}, \mathbf{S}, \mathbf{P}, \mathbf{P}_{U}$.

For $m<\omega$, let $\mathbf{C}_{m}$ be the algebra $\left(\mathbf{C}_{\infty}\right)_{a^{m}}$; i.e.,

$$
\mathbf{C}_{m}=\left\langle\left\{1, a, a^{2}, \ldots, a^{m}\right\}, \cdot a^{m}, \rightarrow, 1\right\rangle .
$$

Since $\mathbf{C}_{m}$ is a bounded Wajsberg hoop, it is a reduct of the Wajsberg algebra

$$
\mathbf{W} \mathbf{a}_{m}=\left\langle\left\{1, a, a^{2}, \ldots, a^{m}\right\}, \cdot_{a^{m}}, \rightarrow, a^{m}, 1\right\rangle .
$$

Chang [6] showed that the variety of Wajsberg algebras is generated by the algebras $\mathbf{W} \mathbf{a}_{m}: \mathbb{W} \mathbb{A}=\mathbf{H S P}\left\{\mathbf{W} \mathbf{a}_{m}: m<\omega\right\}$. In particular, the variety is generated by its finite members. From this it is easily seen, using Proposition 2.2, that a similar statement holds for Wajsberg hoops: $\mathbb{W H O}=\mathbf{H S P}\left\{\mathbf{C}_{m}: m<\omega\right\}$. In the sequel we will need the stronger result that these varieties are actually generated 
by their finite members as a quasivariety. For the class of Wajsberg algebras, the question whether this is so was raised as an open problem in [10] $\left(^{1}\right)$. The following theorem, which appears in Büchi and Owens' manuscript ([5]) without satisfactory proof, is the key.

Theorem 2.5. Let $\mathbf{A}$ be a totally ordered Wajsberg hoop and $\mathbf{C}$ a finite partial subalgebra of $\mathbf{A}$. Then $\mathbf{C}$ is isomorphic to a partial subalgebra of one of the $\mathbf{C}_{m}$, $m=1,2,3, \ldots$

Pro of (Sketch). We use the fact every totally ordered Wajsberg hoop can be embedded in a Wajsberg hoop of the form $\mathbf{P}(\mathbf{G})_{a}$, for some totally ordered Abelian $l$-group $\mathbf{G}$, and some $a \in P(\mathbf{G})$. Now with the finite partial subalgebra $\mathbf{C}$ of the Wajsberg hoop $\mathbf{P}(\mathbf{G})_{a}$ we can associate a universal Horn sentence $\phi_{\mathbf{C}}$ in the language of hoops such that for any totally ordered Abelian $l$-group $\mathbf{H}$, the sentence $\phi_{\mathbf{C}}$ holds in $\mathbf{P}(\mathbf{H})$ if and only if for no $x \in P(\mathbf{H}), \mathbf{C}$ is embeddable in $\mathbf{P}(\mathbf{H})_{x}$. In particular, the sentence $\phi_{\mathbf{C}}$ is not true in $\mathbf{P}(\mathbf{G})$. Using Corollary 2.4 we see that $\mathbf{P}(\mathbf{G}) \in \mathbf{I} \mathbf{S} \mathbf{P}_{U}\left(\mathbf{C}_{\infty}\right)$, hence the sentence $\phi_{\mathbf{C}}$ cannot hold in $\mathbf{P}(\mathbf{Z}) \cong \mathbf{C}_{\infty}$ either. We conclude that there is an $a \in C_{\infty}$ such that $\mathbf{C}$ can be embedded as a partial algebra in $\left(\mathbf{C}_{\infty}\right)_{a}$, i.e., in some $\mathbf{C}_{m}$.

An analogous result holds for Wajsberg algebras.

COROLLARY 2.6. (1) The variety of Wajsberg hoops is generated, as a quasivariety, by its finite algebras; in fact, $\mathbb{W H O}=\mathbf{I S P P}_{U}\left\{\mathbf{C}_{m}: m<\omega\right\}$.

(2) The variety of Wajsberg algebras is generated, as a quasivariety, by its finite algebras; in fact, $\mathbb{W A}=\mathbf{I S P P}_{U}\left\{\mathbf{W} \mathbf{a}_{m}: m<\omega\right\}$.

3. The structure of subdirectly irreducible hoops. Given two hoops A and $\mathbf{B}$ we can form a new hoop $\mathbf{A} \oplus \mathbf{B}$, roughly speaking by replacing the 1-element of $\mathbf{A}$ by the algebra $\mathbf{B}$. Indeed, if for simplicity we assume $A \cap B=\{1\}$, we define $\mathbf{A} \oplus \mathbf{B}$ to be the algebra with domain $A \cup B$, and operations $1^{\mathbf{A} \oplus \mathbf{B}}=1^{\mathbf{A}}=1^{\mathbf{B}}$, and

$$
x \rightarrow y= \begin{cases}x \rightarrow^{\mathbf{A}} y & \text { for } x, y \in A \\ x \rightarrow^{\mathbf{B}} y & \text { for } x, y \in B \\ y & \text { for } x \in B, y \in A, \\ 1 & \text { for } x \in A \backslash\{1\}, y \in B\end{cases}
$$

and

$$
x \cdot y= \begin{cases}x \cdot{ }^{\mathbf{A}} y & \text { for } x, y \in A, \\ x \cdot{ }^{\mathbf{B}} y & \text { for } x, y \in B, \\ y & \text { for } x \in B, y \in A \backslash\{1\}, \\ x & \text { for } x \in A \backslash\{1\}, y \in B .\end{cases}
$$

The algebra $\mathbf{A} \oplus \mathbf{B}$ is a hoop and will be called the ordinal sum of $\mathbf{A}$ and $\mathbf{B}$. Observe that $\mathbf{A}$ and $\mathbf{B}$ are subalgebras of $\mathbf{A} \oplus \mathbf{B}$, that $B$ is a filter of $\mathbf{A} \oplus \mathbf{B}$, and

$\left({ }^{1}\right)$ The result is actually implicit in Wójcicki's work on consequence relations extending the one associated with Łukasiewicz's $\aleph_{0}$-valued logic; see [16]. 
that $\mathbf{A} \oplus \mathbf{B} / B \cong \mathbf{A}$. Finally, since for any two hoops $\mathbf{A}, \mathbf{B}$ we have $\mathbf{A} \cong \mathbf{1}^{\mathbf{B}} \times \mathbf{A}$, and $\mathbf{B} \cong \mathbf{B} \times \mathbf{1}^{\mathbf{A}}$, and the intersection of the universes of these two algebras equals the 1-element of each, we can define $\mathbf{A} \oplus \mathbf{B}$ to be the ordinal sum of these two algebras in case $A \cap B \neq\{1\}$.

If $\mathbf{A}$ and $\mathbf{B}$ are hoops and furthermore $\mathbf{B}$ is subdirectly irreducible, then $\mathbf{A} \oplus \mathbf{B}$ is subdirectly irreducible as well. Conversely, we will show that if $\mathbf{A}$ is a subdirectly irreducible hoop, then $\mathbf{A}=\mathbf{B} \oplus \mathbf{C}$, for some hoop $\mathbf{B}$ and some subdirectly irreducible hoop $\mathbf{C}$. In fact, $\mathbf{C}$ can even be assumed to be a subdirectly irreducible Wajsberg hoop. We will now indicate how this decomposition of a subdirectly irreducible hoop is obtained.

Let $\mathbf{A}$ be a subdirectly irreducible hoop, with minimal filter $U$. We say that $a \in A$ is fixed if for all $u \in U, u \rightarrow a=a$. Let $F$ be the set of fixed elements of A, and let $S$, the support of $U$, be the set $(A \backslash F) \cup\{1\}$. We have

Theorem 3.1. Let $\mathbf{A}$ be a subdirectly irreducible hoop, with $U, F$ and $S$ as above.

(1) $U, F$ and $S$ are sub-universes of $\mathbf{A}$,

(2) $U \subseteq S$, and $\mathbf{S}$ is a subdirectly irreducible Wajsberg hoop; in particular, $\mathbf{S}$ is totally ordered,

(3) $\mathbf{A}=\mathbf{F} \oplus \mathbf{S}$.

The theorem may be seen as a generalization of the the well-known characterization of the subdirectly irreducible Brouwerian semilattices as the algebras $\mathbf{A}$ that can be written $\mathbf{B} \oplus \mathbf{C}_{1}$, for some Brouwerian semilattice $\mathbf{B}$; recall that $\mathbf{C}_{1}$ is the 2-element hoop, which of course is a reduct of the 2-element Boolean algebra. If here $e$ denotes the element of $A$ with the property $e<1$ and for all $x \in A$ either $x=1$ or $x \leq e$ then we have $U=S=\{e, 1\}$, and $F=A \backslash\{e\}$. On the other hand, in case $\mathbf{A}$ is the simple Wajsberg hoop $\mathbf{C}_{m}$, we have $U=S=C_{m}$, and $F=\{1\}$. In general, however, we should think of a subdirectly irreducible hoop as suggested in Figure 3.1.

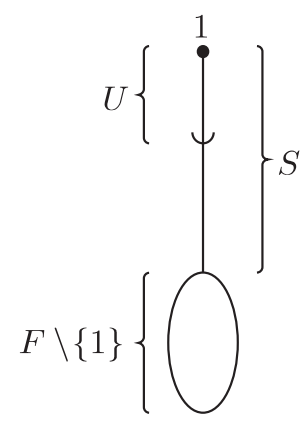

Fig. 3.1. A subdirectly irreducible hoop

The idea of decomposing a subdirectly irreducible hoop as an ordinal sum of a hoop and a Wajsberg hoop is due to Büchi and Owens; our contribution here 
consists in providing a proof that this indeed can always be done. An important role in our argument is played by the identity

(L) $\quad(x \rightarrow y) \rightarrow(y \rightarrow x) \approx(y \rightarrow x)$.

It can be shown to hold in $\mathbb{H} \mathbb{O}$, and yields easily the claim that $\mathbf{S}$ is linearly ordered.

For a variety $\mathbb{V}$ of Wajsberg hoops we define a natural sequence of varieties $\mathbb{V}^{n}, n<\omega:$

(i) $\mathbb{V}^{0}$ is the trivial variety of hoops,

(ii) $\mathbb{V}^{n+1}=\mathbf{H S P}\left(\left(\mathbb{V}^{n}\right)^{+}\right)$, where $\left(\mathbb{V}^{n}\right)^{+}$is the class of algebras $\mathbf{B} \oplus \mathbf{C}$ such that $\mathbf{B} \in \mathbb{V}^{n}$ and $\mathbf{C} \in \mathbb{V}, \mathbf{C}$ totally ordered.

Lemma 3.2. Let $\mathbf{A} \in \mathbb{H} \mathbb{O}$ be subdirectly irreducible and n-generated for some $n<\omega$. Then $\mathbf{A} \in(\mathbb{W} \mathbb{H} \mathbb{O})^{n}$.

Proof. We will use $U, \mathbf{F}$ and $\mathbf{S}$ as above. By Theorem 3.1 we may assume $\mathbf{A}=\mathbf{F} \oplus \mathbf{S}$. Observe that since $U \subseteq S,|S|>1$. Let $X$ be a set of generators of $\mathbf{A}$; we may assume $|X|=n$. Since $F$ is a subuniverse of $A$ we have $X \cap(S \backslash\{1\}) \neq \emptyset$. Since $\mathbf{A} / S \cong \mathbf{F}$, we see that $F$ is therefore $(n-1)$-generated. An easy induction now completes the proof.

We conclude:

THEOREM 3.3. $\mathbb{H O}=\mathbf{I S P P}_{U}\left(\bigcup_{n=0}^{\infty}(\mathbb{W} \mathbb{H} \mathbb{O})^{n}\right)$, and hence $\mathbb{H} \mathbb{O}=\bigvee_{n=0}^{\infty}(\mathbb{W} \mathbb{H} \mathbb{O})^{n}$, where the join is taken in the lattice of subquasivarieties of $\mathbb{H} O$.

Let $\mathbb{B S}$ denote the variety of Brouwerian semilattices, and $\mathbb{B H}(\mathbb{O}$ the variety $\operatorname{HSP}\left(\mathbf{C}_{1}\right)$ of Boolean hoops generated by the two-element Boolean hoop. It is well known that $\mathbb{B S}=\bigvee_{n=0}^{\infty}(\mathbb{B H} \mathbb{O})^{n}$. It is in this sense that the class of hoops relates to the class of Wajsberg algebras as does the class of Brouwerian semilattices to the class of Boolean hoops. Phrased in terms of deductive systems, the class of hoops is an algebraic semantics of an algebraizable deductive system that relates to Łukasiewicz's many-valued logic just like the intuitionistic propositional calculus relates to the classical propositional calculus - at least as far as their respective $\{\cdot, \rightarrow, 1\}$-fragments are concerned.

To conclude this section we show that the variety of hoops is generated, as a quasivariety, by its finite members. By the last theorem, it will suffice to show this for the varieties $(\mathbb{W} \mathbb{H} \mathbb{O})^{n}, n<\omega$.

Theorem 3.4. Let $\mathbf{A} \in\left(\mathbb{W} \mathbb{H}(\mathbb{O})^{n}, n<\omega\right.$, and let $\mathbf{P}$ be a finite partial subalgebra of $\mathbf{A}$. Then there is a finite algebra $\mathbf{A}^{\prime} \in(\mathbb{W H O})^{n}$ in which $\mathbf{P}$ can be embedded.

P r o of. We prove the claim of the theorem by induction on $n$.

For $n=0$ the claim is trivially true. To prove the claim of the theorem for $n \geq 1$, first observe, using Jónsson's Lemma, that every subdirectly irreducible algebra in $(\mathbb{W} \mathbb{H} \mathbb{O})^{n}$ belongs to $\left((\mathbb{W} \mathbb{H} \mathbb{O})^{n-1}\right)^{+}$. It will therefore suffice to show 
that if $\mathbf{P}$ is a partial subalgebra of $\mathbf{A}=\mathbf{B} \oplus \mathbf{C}$, with $\mathbf{B} \in\left(\mathbb{W} \mathbb{H}(\mathbb{O})^{n-1}\right.$ and $\mathbf{C}$ a totally ordered Wajsberg hoop, then there is a finite algebra $\mathbf{B}^{\prime} \in(\mathbb{W H O})^{n-1}$ and an $m<\omega$ such that $\mathbf{P}$ can be embedded in $\mathbf{B}^{\prime} \oplus \mathbf{C}_{m}$. Now let $\mathbf{P}_{1}$ be the partial subalgebra of $\mathbf{P}$ and of $\mathbf{B}$ with universe $B \cap P$. By the induction hypothesis, $\mathbf{P}_{1}$ can be embedded in a finite algebra $\mathbf{B}^{\prime} \in(\mathbb{W} \mathbb{H} \mathbb{O})^{n-1}$. Let $\mathbf{P}_{2}$ be the partial subalgebra of $\mathbf{C}$ and $\mathbf{P}$ with domain $C \cap P$. Since $\mathbf{C}$ is a totally ordered Wajsberg hoop it follows from Theorem 2.5 that $\mathbf{P}_{2}$ can be embedded in one of the hoops $\mathbf{C}_{m}, m<\omega$. It is now not difficult to see that $\mathbf{P}$ itself can be embedded in $\mathbf{B}^{\prime} \oplus \mathbf{C}_{m} \in(\mathbb{W} \mathbb{H} \mathbb{O})^{n}$.

COROLlary 3.5. The varieties $(\mathbb{W H} \mathbb{O})^{n}, n<\omega$, are generated, as quasivarieties, by their finite members; in fact, for $n<\omega$,

$$
(\mathbb{W H O})^{n+1}=\mathbf{I S P P}_{U}\left\{\mathbf{B} \oplus \mathbf{C}_{m}: \mathbf{B} \text { finite, } \mathbf{B} \in(\mathbb{W} \mathbb{H} O)^{n}, m<\omega\right\} \text {. }
$$

COROLlary 3.6. The variety of hoops is generated, as a quasivariety, by its finite members. Hence its quasi-equational theory is decidable.

The last corollary may be seen as the main result of the paper. A weaker version - saying that the variety of hoops is generated by its finite members - was formulated by Büchi and Owens in their manuscript, and the overall strategy of our proof can be found there as well. Our contributions to the proof consist in filling some gaps, by providing a proof of Theorem 2.5, and correcting the construction and proof of Theorem 3.1. Our methods also allowed us to obtain the sharper quasivariety version of the result.

4. The implicational reducts of hoops. We already observed in Section 1 that if $\mathbf{A}=\langle A, \cdot, \rightarrow, 1\rangle$ is a hoop, then $\langle A, \rightarrow, 1\rangle$ is a BCK-algebra satisfying the axiom $(\mathrm{H}):(x \rightarrow y) \rightarrow(x \rightarrow z) \approx(y \rightarrow x) \rightarrow(y \rightarrow z)$. We denote the class of all BCK-algebras satisfying axiom $(\mathrm{H})$ by $\mathbb{H} \mathbb{B} \mathbb{C K}$. In this section we will show that the quasivariety of $\{\rightarrow, 1\}$-subreducts of hoops is precisely $\mathbb{H} \mathbb{B} \mathbb{C}$, and that furthermore it is a variety generated, as a quasivariety, by its finite algebras.

First we will concentrate on the $\{\rightarrow, 1\}$-subreducts of Wajsberg hoops, the class which figured so prominently in the description of the subdirectly irreducible hoops. These $\{\rightarrow, 1\}$-subreducts are BCK-algebras that not only satisfy $(\mathrm{H})$, but also the commutative law (T) (see Section 2) and the identity (L) (see Section 3). Such BCK-algebras are known as Eukasiewicz BCK-algebras, and we will denote this class - that actually can be axiomatized by the BCK-axioms together with (T) and (L) - by $\mathbb{L} \mathbb{B} \mathbb{C}$. Observe that the quasi-identity (M7) can be derived from the identities (M4) and (T), and therefore can be omitted from the axioms of $\mathbb{L} \mathbb{B C K}$; it follows that $\mathbb{L} \mathbb{B} \mathbb{C}$ is a variety. It is not difficult to see that the subdirectly irreducible algebras in $\mathbb{L} \mathbb{B} \mathbb{C} K$ are totally ordered; see [13] for a description of the subvarieties of $\mathbb{L} \mathbb{B} \mathbb{C}$.

It is well known that if $\mathbf{A}=\langle A, \rightarrow, 1\rangle$ is a bounded totally ordered commutative BCK-algebra, then in fact it is a reduct of a Wajsberg algebra. Indeed, if 0 is the 
(lower) bound of $\mathbf{A}$, and we define

$$
\neg x:=x \rightarrow 0, \quad x \cdot y:=\neg(x \rightarrow \neg y),
$$

then $\langle A, \cdot, \rightarrow, 0,1\rangle$ is a Wajsberg algebra. The following analog of Lemma 2.1 is easy to establish. Here, for a BCK-algebra $\mathbf{A}=\langle A, \rightarrow, 1\rangle$, and $a \in A, \mathbf{A}_{a}$ will denote the subalgebra of $\mathbf{A}$ with domain $A_{a}=\{x \in A: x \geq a\}$.

Lemma 4.1. Let $\mathbf{A}$ be a (downward) directed BCK-algebra, i.e., for all $x, y \in A$ there is a $z \in A$ such that $z \leq x, z \leq y$. Then $\mathbf{A} \in \mathbf{I S P}_{U}\left(\left\{\mathbf{A}_{a}: a \in A\right\}\right)$.

In particular, every totally ordered commutative BCK-algebra can be embedded into a bounded totally ordered commutative BCK-algebra, and is therefore isomorphic to a subreduct of a Wajsberg algebra. Since of course every Wajsberg algebra is also a Wajsberg hoop we have:

Proposition 4.2. The class of $\{\rightarrow, 1\}$-subreducts of Wajsberg hoops coincides with the variety $\mathbb{L} \mathbb{B} \mathbb{C}$ of Lukasiewicz BCK-algebras: $\mathbf{S}(\mathbb{W} \mathbb{H} \mathbb{O}\{\rightarrow, 1\})=\mathbb{L} \mathbb{B} \mathbb{C}$.

A description of the subdirectly irreducibles of $\mathbb{H} \mathbb{B C K}$ similar to that of the subdirectly irreducible hoops obtained in Theorem 3.1 will enable us to lift the result that every algebra in $\mathbb{L} \mathbb{B} \mathbb{C} \mathbb{K}$ can be embedded in the reduct of a Wajsberg hoop to the result we are aiming for, that every algebra in $\mathbb{H} \mathbb{B C K}$ can be embedded in a hoop. One slight complication we have to deal with is that we don't know whether $\mathbb{H B \mathbb { C }}$ is a variety of BCK-algebras; we will therefore have to resort to using notions relativized to the quasivariety $\mathbb{B} \mathbb{C}$. If $\mathbf{A} \in \mathbb{B} \mathbb{C}$ then a congruence $\Theta$ of $\mathbf{A}$ is called a relative congruence if $\mathbf{A} / \Theta \in \mathbb{B} \mathbb{C}$. A subset $F$ of the BCK-algebra $\mathbf{A}$ is a filter of $\mathbf{A}$ if $1 \in F$ and $x, x \rightarrow y \in F$ imply $y \in F$. The relative congruences of $\mathbf{A}$ correspond naturally to the filters of $\mathbf{A}$ : the map $\Theta \mapsto 1 / \Theta$ establishes a 1-1 correspondence between relative congruences of $\mathbf{A}$ and filters of $\mathbf{A}$, with inverse $F \mapsto\{(x, y): x \rightarrow y \in F, y \rightarrow x \in F\}$. In fact, this 1-1 correspondence is an isomorphism between the algebraic lattice of all relative congruences of $\mathbf{A}$ and the lattice of filters of $\mathbf{A}$. An algebra $\mathbf{A} \in \mathbb{B} \mathbb{C K}$ is a relatively subdirectly irreducible algebra if it possesses a filter $U \neq\{1\}$ with the property that for all filters $F$ of $\mathbf{A}$, if $F \neq\{1\}$, then $U \subseteq F$. Every BCK-algebra is a subdirect product of relatively subdirectly irreducible BCK-algebras. Since $\mathbb{H} \mathbb{B} \mathbb{C K}$ is defined, relative to $\mathbb{B C K}$, by an identity, every algebra $\mathbf{A} \in \mathbb{H} \mathbb{B} \mathbb{C K}$ is a subdirect product of relatively subdirectly irreducible algebras in $\mathbb{H B C} \mathbb{K}$.

Now we come to the crucial step in our proof, viz. the analog of Theorem 3.1 for the class $\mathbb{H B C K}$. The proof closely parallels that of Theorem 3.1, and is obtained by casting all arguments in a form involving $\rightarrow$ only. It was carried out by the second author solely, as part of her thesis work (for the details see [8]).

We define the notion of ordinal sum $\oplus$ for BCK-algebras perfectly analogous to the ordinal sum of hoops, as in Section 3, by just ignoring the clauses involving the product operation. It is easy to see that if $\mathbf{A}, \mathbf{B} \in \mathbb{H} \mathbb{B} \mathbb{K}$, then $\mathbf{A} \oplus \mathbf{B} \in \mathbb{H} \mathbb{B} \mathbb{K}$. 
Again, if $A \cap B=\{1\}$, then $B$ is a filter of $\mathbf{A} \oplus \mathbf{B}$ and $\mathbf{A} \oplus \mathbf{B} / B \cong \mathbf{A}$. If in addition $\mathbf{B}$ is relatively subdirectly irreducible, then so is $\mathbf{A} \oplus \mathbf{B}$.

Now suppose $\mathbf{A} \in \mathbb{H} \mathbb{B} \mathbb{C}$ is relatively subdirectly irreducible, with minimal filter $U \neq\{1\}$. As before, call $a \in A$ a fixed element of $\mathbf{A}$ if for all $u \in U$, $u \rightarrow a=a$. Let $F$ be the set of all fixed elements of $\mathbf{A}$, and let $S$ be the set $(A \backslash F) \cup\{1\}$. We have:

TheOREM 4.3. Let $\mathbf{A} \in \mathbb{H} \mathbb{B} \mathbb{C} K$ be relatively subdirectly irreducible, with $U, F$ and $S$ as above. Then

(1) $U, F$ and $S$ are subuniverses of $\mathbf{A}$,

(2) $U \subseteq S$, and $\mathbf{S}$ is a subdirectly irreducible Eukasiewicz BCK-algebra; in particular, $\mathbf{S}$ is totally ordered,

(3) $\mathbf{A}=\mathbf{F} \oplus \mathbf{S}$.

This result enables us to obtain analogs of the results on hoop varieties obtained in Section 3. We will restrict ourselves here to our stated goal, to showing $\mathbb{H B C K}$ is precisely the class of $\{\rightarrow, 1\}$-subreducts of hoops.

Theorem 4.4. Let $\mathbf{A}$ be a finitely generated algebra in $\mathbb{H B C K}$. Then $\mathbf{A}$ is isomorphic to a subreduct of a hoop.

Pr o of. We induct on the number $n$ of generators of $\mathbf{A}$. If $n=0$, then $\mathbf{A}$ is the 1-element algebra, and hence a reduct of a hoop. Now assume the claim holds for all algebras in $\mathbb{H} \mathbb{B C K}$ that are generated by $n$ or fewer elements, for some $n<\omega$. Suppose that $\mathbf{A} \in \mathbb{H} \mathbb{B} \mathbb{C K}$ is $(n+1)$-generated. Clearly it will suffice to prove $\mathbf{A}$ is isomorphic to a subreduct of a hoop under the additional assumption $\mathbf{A}$ is relatively subdirectly irreducible. A can then be written as $\mathbf{F} \oplus \mathbf{S}$, with $\mathbf{F}$ and $\mathbf{S}$ as before. As in the proof of Theorem 3.4, we see $\mathbf{F}$ is $n$-generated. By the induction hypothesis $\mathbf{F}$ is isomorphic to a subreduct of a hoop $\mathbf{F}^{\prime}$. Since $\mathbf{S} \in \mathbb{L} \mathbb{B} \mathbb{C}$, by Proposition $4.2 \mathbf{S}$ is isomorphic to a subreduct of a hoop as well, say of $\mathbf{S}^{\prime}$, with $\mathbf{S}^{\prime} \in \mathbb{L B} \mathbb{C}$. But then $\mathbf{A}$ is isomorphic to a subreduct of the hoop $\mathbf{F}^{\prime} \oplus \mathbf{S}^{\prime}$.

Since any algebra can be embedded in an ultraproduct of its finitely generated substructures we can now confirm a conjecture by Wroński ([17]):

Corollary 4.5. The class of $\{\rightarrow, 1\}$-subreducts of hoops coincides with the class $\mathbb{H} \mathbb{B} \mathbb{C K}: \mathbf{S}(\mathbb{H} \mathbb{O}\{\rightarrow, 1\})=\mathbb{H B \mathbb { C } K}$.

Using the same method of proof the following relativized version of this result can be obtained. Let $x \rightarrow^{0} y:=y$, and for $n<\omega, x \rightarrow^{n+1} y:=x \rightarrow\left(x \rightarrow^{n} y\right)$. For $2 \leq n<\omega$ let $\varepsilon_{n}$ denote the identity $x \rightarrow^{n} y \approx x \rightarrow^{n-1} y$. It is easy to see that this identity is satisfied in a hoop $\mathbf{A}$ if and only if it is n-potent, i.e., satisfies the identity $x^{n} \approx x^{n-1}$. Let $\mathbb{H} \mathbb{O}(n)$ denote the class of $n$-potent hoops; in particular, $\mathbb{H} \mathbb{O}(2)$ is the variety of Brouwerian semilattices. In [3, Section 7] the problem was raised whether every algebra in $\mathbb{H} \mathbb{B} \mathbb{C}$ satisfying $\varepsilon_{n}$ is isomorphic to a subreduct of an algebra in $\mathbb{H O}(n)$. It is well known that for $n=2$ the answer 
is affirmative: indeed, every Hilbert algebra is a subreduct of some Brouwerian semilattice. More generally we have:

THEOREM 4.6. The class of $\{\rightarrow, 1\}$-subreducts of n-potent hoops coincides with the variety of algebras in $\mathbb{H} \mathbb{B} \mathbb{C K}$ satisfying the identity $\varepsilon_{n}$, for $2 \leq n<\omega$.

For the proof we refer to [8].

In the above theorem, the presence of the identity $\varepsilon_{n}$ allows us to drop the quasi-identity (M7) from the axioms, and to conclude the class is a variety. We conclude this section by demonstrating the quasivariety $\mathbb{H} \mathbb{B} \mathbb{C}$ itself is a variety as well.

Cornish introduced in $[7]$ an identity he called $(\mathrm{J})$ :

$(\mathrm{J}) \quad(((x \rightarrow y) \rightarrow y) \rightarrow x) \rightarrow x \approx(((y \rightarrow x) \rightarrow x) \rightarrow y) \rightarrow y$.

Let $\mathbb{B} \mathbb{B} \mathbb{K}$ denote the class of BCK-algebras satisfying the identity $(\mathrm{J})$. Again it is not difficult to see the identity $(\mathrm{J})$ makes the quasi-identity (M7) superfluous among the axioms of BCK-algebras, and that hence $\mathbb{J B C K}$ is a variety of BCKalgebras. It is also easy to see that every commutative BCK-algebra satisfies $(\mathrm{J})$, and hence, in particular, that $\mathbb{L} \mathbb{B} \mathbb{C} \subseteq \mathbb{J} \mathbb{B} \mathbb{K}$. Finally, $\mathbb{I} \mathbb{B} \mathbb{K}$ is closed under ordinal sums. The proof of the following theorem now follows the lines of that of Theorem 4.4:

Theorem 4.7. Let $\mathbf{A} \in \mathbb{H} \mathbb{B} \mathbb{C} K$ be n-generated, for $n<\omega$. Then $\mathbf{A} \in \mathbb{J} \mathbb{B} \mathbb{C K}$.

COROLlary 4.8. Every algebra in $\mathbb{H B \mathbb { B }}$ satisfies identity $(\mathrm{J})$, and therefore $\mathbb{H} \mathbb{B C K}$ can be axiomatized by the identities (M3), (M4), (M5), (M8), (M9), (H) and $(\mathrm{J})$. The class $\mathbb{H} \mathbb{B} \mathbb{C}$ is thus a variety.

This solves a problem proposed in [17]. We have not been able to find a syntactic derivation of the identity $(\mathrm{J})$ from the axioms of $\mathbb{H} \mathbb{B} \mathbb{C} K$.

\section{References}

[1] G. Birkhoff, Lattice Theory, Amer. Math. Soc. Colloq. Publ. 25, 3rd ed., Amer. Math. Soc., Providence 1967.

[2] W. J. Blok and D. Pigozzi, Algebraizable Logics, Mem. Amer. Math. Soc. 396 (1989).

[3] - - - On the structure of varieties with equationally definable principal congruences III, Algebra Universalis, to appear.

[4] B. Bosbach, Komplementäre Halbgruppen. Kongruenzen und Quotienten, Fund. Math. 64 (1970), 1-14.

[5] J. R. Büchi and T. M. Owens, Complemented monoids and hoops, unpublished manuscript.

[6] C. C. Chang, A new proof of the completeness of the Eukasiewicz axioms, Trans. Amer. Math. Soc. 93 (1959), 74-80.

[7] W. H. Cornish, A large variety of BCK-algebras, Math. Japon. 26 (1981), 339-342.

[8] I. M. A. Ferreirim, On varieties and quasivarieties of hoops and their reducts, thesis, Univ. of Illinois at Chicago, 1992. 
[9] I. Fleischer, Every BCK-algebra is a set of residuables in an integral pomonoid, J. Algebra 119 (1988), 360-365.

[10] H. Gaitan, Quasivarieties of Wajsberg algebras, J. Non-Classical Logic 8 (1991), 79-101.

[11] D. Higgs, Dually residuated commutative monoids with identity element do not form an equational class, Math. Japon. 29 (1984), 69-75.

[12] W. C. Holland, A. H. Mekler, and N. R. Reilly, Varieties of lattice-ordered groups in which prime powers commute, Algebra Universalis 23 (1986), 196-214.

[13] Y. Komori, Super-Eukasiewicz implicational logics, Nagoya Math. J. 72 (1978), 127-133.

[14] H. Ono and Y. Komori, Logics without the contraction rule, J. Symbolic Logic 50 (1985), 169-201.

[15] M. Pałasiński, An embedding theorem for BCK-algebras, Math. Seminar Notes Kobe Univ. 10 (1982), 749-751

[16] R. Wójcicki, On matrix representations of consequence operations of Eukasiewicz's sentential calculi, Z. Math. Logik Grundlag. Math. 19 (1973), 239-247.

[17] A. Wroński, An algebraic motivation for BCK-algebras, Math. Japon. 30 (1983), 187193

[18] —, BCK-algebras do not form a variety, ibid. 28 (1983), 211-213. 